\title{
ON THE CHERN CLASSES OF THE REGULAR REPRESENTATIONS OF SOME FINITE GROUPS
}

\author{
by BENJAMIN M. MANN and R. JAMES MILGRAM* \\ (Received 10th February 1981)
}

In studying the cohomology of the symmetric groups and its applications in topology one is led to certain questions concerning the representation rings of special subgroups of $\mathscr{S}_{n}$. In this note we calculate the Chern classes of the regular representation of $(Z / p)^{n}$ where $p$ is a fixed odd prime in terms of certain modular invariants first described by $L$. E. Dickson in 1911. In a later paper [9] we apply these results to study the odd primary torsion in the $P L$ cobordism ring. Some indications of this application are given in Sections 10-12 where we apply the result above to obtain information about the cohomology of $\mathscr{S}_{p^{n}}$. After circulation of this note in preprint form we learned that $\mathrm{H}$. Mui [10], has also proved Theorem 6.2.

1.

Recall that the regular representation $R(G)$ of a finite group $G$ is obtained via its natural action as a group of automorphisms of the group ring $C(G)\left(g \Sigma z_{i} g_{i}=\Sigma z_{i} g g_{i}\right)$. In particular, from the theory of representations of finite groups [3] we have the decomposition

$$
R(G)=\sum_{S(G)}(\operatorname{dim} S(G)) S(G)
$$

where $S(G)$ runs over all irreducible complex representations of $G$.

In the case $G=(Z / p)^{n}$ all the irreducible representations are one dimensional and are obtained explicitly as follows. Let

$$
\lambda: Z / p \rightarrow U(1)
$$

be the homomorphism defined by

$$
\lambda(T)=e^{(2 \pi i / p)}
$$

where $T$ is a specified generator of $Z / p$. Then to each $n$-tuple $I=\left(i_{1} \ldots i_{n}\right) \in(Z / p)^{n}$ define

$$
V_{I}:(Z / p)^{n} \rightarrow Z / p
$$

*This research was supported in part by the NSF GP Grant 4.3967. 
as the homomorphism given on the $j$ th generator $T_{j}$ by

$$
V_{I}\left(T_{j}\right)=T^{(i j)} .
$$

The composites

$$
\lambda \circ V_{I}=S_{I}:(Z / p)^{n} \rightarrow U(1)
$$

are irreducible, and indeed give all the irreducible representations of $(Z / P)^{n}$.

2.

The cohomology of $(Z / p)^{n}$ is given by

$$
H^{*}\left((Z / p)^{n}, Z / p\right)=E\left(e_{1} \ldots e_{n}\right) \otimes P\left(b_{1}, \ldots, b_{n}\right)
$$

where $\operatorname{dim}\left(e_{i}\right)=1, \operatorname{dim}\left(b_{i}\right)=2$. Let $I_{i} \operatorname{denote}(0 \ldots 010 \ldots 0)$ then

$$
e_{i}=V_{I_{i}}^{*} e
$$

and

$$
b_{i}=V_{I_{i}}^{*} b
$$

with $e, b$, the canonical generators of $H^{*}(Z / p, Z / p)=E(e) \otimes P(b)$.

It is well known that the homomorphism $\lambda_{i}: Z / p \rightarrow Z / p$ defined by $\lambda_{i}(T)=T^{i}$ induces the cohomology map $\left(\lambda_{i}\right)^{*}(e)=i e,\left(\lambda_{i}\right)^{*}(b)=i b$.

From this and the Künneth theorem it is easy to see that

$$
\begin{aligned}
& V_{(1,1, \ldots, 1)}^{*}(e)=\Sigma\left(e_{i}\right) \\
& V_{(1,1, \ldots, 1)}^{*}(b)=\Sigma\left(b_{i}\right)
\end{aligned}
$$

(Indeed $b$ is the Bochstein of $e$ so it suffices to check on $e$, and here use the maps

$$
\begin{gathered}
\varphi_{1, j}:(Z / p) \rightarrow(Z / p)^{n} \quad \text { defined by } \\
\varphi_{1 j}(T)=T_{1} \circ T_{j}^{-1}
\end{gathered}
$$

together with the observation $V_{(1,1, \ldots, 1)} \varphi_{1 j}(T)=1$.)

Lemma 2.3. Let $I=\left(i_{1}, \ldots, i_{n}\right)$ as in Section 1 then

$$
V_{I}^{*}(e)=\Sigma i_{j} e_{j}, \quad V_{I}^{*}(b)=\Sigma i_{j} b_{j} .
$$


Proof. Let $\varphi_{\left(i_{1} \ldots i_{n}\right)}:(Z / p)^{n} \rightarrow(Z / p)^{n}$ be given by $\varphi_{\left(i_{1} \ldots i_{n}\right)} T_{j}=\left(T_{j}\right)^{i_{j}}$. Then $V_{I}$ is the composite $V_{(1, \ldots, 1)}\left(\varphi_{i_{1} \ldots i_{n}}\right)$. By the Künneth theorem

$$
\begin{aligned}
& \varphi_{\left(i_{1} \ldots i_{n}\right)}^{*}\left(e_{j}\right)=i_{j}\left(e_{j}\right) \\
& \varphi_{\left(i_{1} \ldots i_{n}\right.}^{*}\left(b_{j}\right)=i_{j}\left(b_{j}\right)
\end{aligned}
$$

so 2.3 follows from 2.2 .

3.

The total Chern class of the representation $\lambda$ in (1.2) is $1+b=C(\lambda)$. Moreover, if $R_{1}$ and $R_{2}$ are two representations of $G$ then

$$
C\left(R_{1} \oplus R_{2}\right)=C\left(R_{1}\right) C\left(R_{2}\right)
$$

Thus, we have from (1.1), (2.3)

$$
C\left(R(Z / p)^{n}\right)=\prod_{\left(i_{1} \ldots i_{n}\right) \in(Z / p)^{n}}\left(1+i_{1} b_{1}+\ldots+i_{n} b_{n}\right)
$$

To calculate (3.2) explicitly we introduce

Definition 3.3. The Chern polynomial $C P(R)$ of a representation $R$ of dimension $n$ is the element

$$
C P(R)=\Sigma c_{i}(R) x^{n-i}
$$

in the ring of polynomials in $x$ with coefficients in $H^{*}(G, Z / p)$.

We see directly that

$$
C P\left(R_{1} \oplus R_{2}\right)=C P\left(R_{1}\right) C P\left(R_{2}\right)
$$

so we have

$$
\begin{aligned}
C P\left(R(Z / p)^{n}\right) & =\prod_{\left(i_{1} \ldots i_{n}\right) \in(Z / p)^{n}}\left(x+i_{1} b_{1}+\ldots+i_{n} b_{n}\right) \\
& =x\left(\sum_{\left(i_{1} \ldots i_{n}\right) \neq(0, \ldots, 0)} c_{i}\left(R(Z / p)^{n}\right) x^{p^{n}-i-1}\right) \\
& =C P\left(R(Z / p)^{n}-1\right) \circ C P(1)
\end{aligned}
$$

where 1 is the trivial representation. 
4.

The general linear group $G L_{n}(Z / p)$ acts on $(Z / p)^{n}$ as its group of automorphisms, and hence by composition on the representation ring, $(\alpha(S(G))$ is the composite

$$
(Z / p)^{n} \stackrel{a}{\rightarrow}(Z / p)^{n} \stackrel{s}{\rightarrow} U(m)
$$

where $G=(Z / p)^{n}, \alpha \in G L_{n}(Z / p)$, and $S$ is a representation) and $\alpha S_{I}=S_{\alpha(l)}$. Clearly, $R(Z / p)^{n}$ -1 and 1 form a basis for the set of $G L_{n}(Z / p)$ invariant representations of $(Z / p)^{n}$.

The action of $G L_{n}(Z / p)$ also induces an action on $H^{*}\left((Z / p)^{n}, Z / p\right)$ (if $\alpha\left(T_{j}\right)$ $=T_{1}^{i_{j, 1}}, \ldots, T_{n}^{i_{n},}$, then $\alpha^{*}\left(e_{k}\right)=\Sigma i_{s, k} e_{s}$ and similarly for $\left.b_{k}\right)$ and from invariance of $R(Z / p)^{n}$ we have

Lemma 4.1. $c_{i}\left(R(Z / p)^{n}\right) \in P\left(b_{1} \ldots b_{n}\right)^{G L_{n}(Z / p)}$ the invariant subring of $P\left(b_{1} \ldots b_{n}\right)$.

5.

Let $p\left(x_{1} \ldots x_{m}\right)$ be a polynomial in $m$ indeterminants with coefficients in the field $Z / p$. Since the $p$ th power homomorphism is the identity on $Z / p$ we have

$$
\left(p\left(x_{1} \ldots x_{m}\right)\right)^{p}=p\left(x_{1}^{p}, \ldots, x_{m}^{p}\right)
$$

Consequently, the determinant

$$
\left|\begin{array}{cccc}
x_{1}^{p_{1}} & x_{2}^{p_{1}^{i_{1}}} & \ldots & x_{m}^{p_{1}} \\
x_{1}^{p_{1}} & x_{2}^{p_{2}} & \ldots & x_{m}^{p_{2}} \\
- & - & - & - \\
x_{1}^{i_{m}} & x_{2}^{p_{m}^{i_{m}}} & \ldots & x_{m}^{p_{m}^{i_{m}}}
\end{array}\right|=D\left(x_{1} \ldots, x_{m}, p^{i_{1}}, p^{i_{2}}, \ldots, p^{i_{m}}\right)
$$

satisfies

$$
D\left(\alpha\left(x_{1}\right), \ldots, \alpha\left(x_{m}\right), p^{i_{1}}, \ldots, p^{i_{m}}\right)=\operatorname{det}(\alpha) D\left(x_{1}, \ldots, x_{m}, p^{i_{1}}, \ldots, p^{i_{m}}\right)
$$

where $\alpha \in G L_{m}(Z / p)$ and $\alpha\left(x_{i}\right)=x_{i}^{\prime}=\Sigma d_{i j} x_{j}$.

We need the following particular cases, which, we should note are non-zero.

Definition 5.4. $\quad D_{n, j}=D\left(x_{1}, \ldots, x_{n}, 1, p, p^{2}, \ldots, \hat{p}^{j}, \ldots, p^{n}\right)$.

In particular, $D_{n, n}$ is usually written $L$ and $D_{n, 0}=L^{P}$. $L$ has an explicit factorisation first discovered by E. H. Moore in 1896.

Lemma 5.5. (Moore [5])

$$
L=\prod_{\left(j_{1} \ldots j_{n}\right)}\left(j_{1} x_{1}+\ldots+j_{n} x_{n}\right)
$$


where $\left(j_{1} \ldots i_{n}\right)$ runs over all elements in $(Z / p)^{n}$ with first non-zero coefficient equal to one.

Proof. (Compare [1, p. 76]). $L$ is invariant under the special linear group $S L_{n}(Z / p)$ which acts transitively on the non-zero elements of $(Z / p)^{n}$. Hence, since $x_{1}$ is a factor of $L$ it follows that $\alpha\left(x_{1}\right)=j_{1} x_{1}+\ldots+j_{n} x_{n}$ is a factor as well. Hence the product above divides $L$ (the factors are all relatively prime). But both sides have the same degree, hence they differ only up to a constant factor. On the other hand, $x_{1}^{p^{n-1}} x_{2}^{p^{n-2}} \ldots x_{n}$ the diagonal term occurs in both sides only once and each time with coefficient 1.

More generally $x_{1}$ is a factor of $D_{n, j}$ for all $j$ so $L$ is also a factor of $D_{n, j}$ by the argument above and we have

Lemma 5.6. $\quad D_{n, j}=Q_{n, j} L$ where $Q_{n, j}$ is a non-zero polynomial invariant under $G L_{n}(Z / p)$.

6.

Dickson's main result in [1], proved by induction on $n$ (for $n=2$ the method is by Galois theory) specifies the $G L_{n}(Z / p)$ invariants as

Theorem 6.1. (Dickson) $P\left(x_{1} \ldots x_{n}\right)^{G L_{n}(Z / p)}$

$$
=P\left(Q_{n, n-1}, Q_{n, n-2}, \ldots, Q_{n, 0}\right)
$$

(Actually, he showed $P\left(x_{1} \ldots x_{n}\right)^{S L_{n}(Z / p)}=P\left(Q_{n, n-1}, \ldots, Q_{n, 1}, L\right)$, but this gives (6.1) directly.)

Here is our main result.

Theorem 6.2. The Chern polynomial for the regular representation of $(Z / p)^{n}$ is

$$
C P\left(R(Z / p)^{n}\right)=x\left((-1)^{n} \sum_{i=0}^{n-1}(-1)^{i} Q_{n, i}\left(b_{1} \ldots b_{n}\right) x^{p^{i}-1}\right) .
$$

The proof decomposes into three steps. First, we note that (6.2) is true up to some undetermined coefficients in $Z / p$. Next, we verify that

$$
c_{p^{n-1}}=(-1)^{n} Q_{n, 0}
$$

These two steps are purely formal algebra. Finally, using the Steenrod $p$ th power operations and $a \bmod (p) \mathrm{Wu}$ formula for the Chern classes we obtain the remaining coefficients and finish the proof.

7.

Lemma 7.1. In degrees less than $p^{n}$ the only elements in $P\left(x_{1} \ldots x_{n}\right)^{G L_{n}(Z / p)}$ are the $Q_{n, j}$. 
Proof. The class of least degree, according to (6.1), is $Q_{n, n-1}$ which has degree $p^{n}$ $-p^{n-1}$ and since

$$
2\left(p^{n}-p^{n-1}\right) \geqq p^{n}>p^{n}-1=\operatorname{dim}\left(Q_{n, 0}\right)
$$

for all $p \geqq 2$ we have (7.1).

Consequently, the only invariants in the range of degrees involved in $C P\left(R(Z / p)^{n}\right)$ are the $Q_{n, i}$ and this gives step 1 .

8.

In (3.4) we set $x=0$ in $C P\left(R(Z / p)^{n}-1\right)$ we obtain

$$
\begin{aligned}
c_{p^{n-1}} & =\prod_{\left(i_{1} \ldots i_{n}\right) \neq 0}\left(i_{1} b_{1}+\ldots+i_{n} b_{n}\right) \\
& =\prod_{k=1}^{n}(p-1) ! !^{n-k} \prod_{\left(0.01, j_{k+1}, \ldots j_{n}\right)}\left(b_{k}+j_{k+1} b_{k+1}+\ldots+j_{n} b_{n}\right)^{p-1} \\
& =(p-1) !{ }^{n} L^{p-1}=(p-1) ! Q_{n, 0} .
\end{aligned}
$$

Now, by Wilson's theorem $(p-1) ! \equiv-1(\bmod p)$ and we have

$$
c_{p^{n-1}}\left(R(Z / p)^{n}-1\right)=c_{p^{n-1}}\left(R(Z / p)^{n}\right)=(-1)^{n} Q_{n, 0}
$$

This is step 2 .

9.

In $H^{*}\left(C P^{\infty}, Z / p\right)=P(b)$ where $b$ is the two dimensional generator, the action of the Steenrod $p$ th power operation $P^{i}$ is given by

$$
P^{i}\left(b^{j}\right)=\left(\begin{array}{l}
j \\
i
\end{array}\right) b^{j+i(p-1)}
$$

In particular, $P^{j}\left(b^{p^{i}}\right)=0$ unless $j=p^{i}$ in which case $p^{p^{i}}\left(b^{p^{i}}\right)=b^{p^{i+1}}$. Using the Cartan formula $P^{i}(x \cup y)=\sum_{j=0}^{i} P^{j}(x) \cup P^{i-j}(y)$ we have the action of $P^{i}$ in $H^{*}\left(\left(C P^{\infty}\right)^{n}, Z / p\right)$ $=P\left(b_{1}, \ldots, b_{n}\right)$.

In particular, the above remarks and (5.3) show

$$
P^{j} D_{n, k}=0 \quad \text { if } \quad 0<j<p^{n} \quad \text { and } \quad j \neq p^{k-1}
$$


but $P^{p^{k-1}} D_{n, k}=D_{n, k-1}$. Thus, we have

Lemma 9.2. $P^{p^{k}}\left(Q_{n, j}\left(b_{1}, \ldots, b_{n}\right)\right)=Q_{n, j-1}\left(b_{1}, \ldots, b_{n}\right)$ if $k=j-1$ and is zero otherwise for $k<n$.

Next, we require

Lemma 9.3. The Chern classes $c_{n}$ reduced $\bmod p$ universally satisfy the Wu formula

$$
P^{i}\left(c_{n}\right)=\left({ }^{n-1}{ }^{-1}\right) c_{n+(p-1) i}+D
$$

where $D$ is a decomposable polynomial in $c_{1}, \ldots, c_{n+(p-1) i-1}$.

Proof. There is a map $f: \Sigma^{2} C P^{\infty} \rightarrow B_{U}$ which satisfies $f^{*}\left(c_{i}\right)=\sigma^{2}\left(b^{i-1}\right)$ for all $i$, and, of course, $f^{*}(D)=0$ for any decomposable. For details see [8, Chapter 4]. Since $P^{i}$ commutes with suspension and with $f^{*},(9.3)$ is a consequence of $(9.1)$.

Thus, we have

$$
p^{p^{r-1}}\left(c_{p^{n}-p^{r}}\right)=\left(p^{n}-p^{r}-1\right) c_{p^{n-p^{r-1}}}=-1 c_{p^{n-p^{r-1}}}
$$

on comparing coefficients in the expansion

$$
(x+1)^{p^{n}-p^{r}-1}=\left(x^{p^{n-1}}+1\right)^{p-1} \ldots\left(x^{p^{r}}+1\right)^{p-2}\left(x^{p^{p-1}}+1\right)^{p-1} \ldots(x+1)^{p-1}
$$

Now using (9.2) and (9.4) together with (8.2) the proof of (6.2) is direct.

10.

If $G$ is a finite group of order $n$ it has a natural embedding $p: G \rightarrow \mathscr{S}_{n}$ obtained by regarding left multiplication by $g \in G$ as a permutation of the elements of $G$. Also, there is the permutation representation of $\mathscr{S}_{n}$

$$
P: \mathscr{P}_{n} \rightarrow U(n)
$$

where $P(\alpha)\left(z_{1} \ldots z_{n}\right)=\left(z_{\alpha^{-1}(1)}, \ldots, z_{\alpha^{-1(n)}}\right)$, and we evidently have

Lemma 10.1. The composite

$$
G \stackrel{p}{\rightarrow} \mathscr{S}_{n} \stackrel{P}{\rightarrow} U(n)
$$

is the regular representation $R(G)$. 
Corollary 10.2. $H^{*}\left(\mathscr{S}_{n}, Z / p\right)$ contains a polynomial subring $\mathscr{P}$ on generators $c_{p^{n} \cdots p^{n}, 1}(P), \ldots, c_{p^{n-1}}(P)$, and $\mathscr{P}$ maps onto

$$
P\left(b_{1}, \ldots, b_{n}\right)^{G L_{n}(Z / p)}
$$

under the induced map

$$
p^{*}: H^{*}\left(\mathscr{P}_{p^{n}}, Z / p\right) \rightarrow H^{*}\left((Z / p)^{n}, Z / p\right)
$$

11.

We need more precise information about the Chern classes of the permutation representation of $\mathscr{S}_{p^{n}}$. To this end consider the inclusion

$$
\left(\mathscr{S}_{p^{n-1}}\right)^{p}\left|\stackrel{i_{n-1}}{\rightarrow}\right| \mathscr{S}_{p^{n}}
$$

where the $p$-tuple $\left(\alpha_{1}, \ldots, \alpha_{p}\right)$ acts by regarding $\alpha_{i}$ as a permutation of the $i$ th block of $p^{n-1}$ letters

$$
\left((i-1) p^{n-1}+1, \ldots, i p^{n-1}\right)
$$

Let $q_{i}\left(\mathscr{S}_{p^{n-1}}\right)^{p} \rightarrow \mathscr{S}_{p^{n-1}}$ be projection onto the $i$ th factor, then by inspection we have

Lemma 11.1. The representation $P_{p^{n}}\left(i_{n-1}\right)$

$$
\begin{gathered}
\left(\mathscr{S}_{p^{n-1}}\right)^{p} \rightarrow U\left(p^{n}\right) \quad \text { is } \\
\sum_{i=1}^{p} P_{p^{n-1}}\left(q_{i}\right) .
\end{gathered}
$$

Consequently, under the composite map

$$
\varphi_{n, 1}:\left[(Z / p)^{n-1}\right]^{p} \stackrel{p \times \ldots \times p}{\longrightarrow}\left(\mathscr{S}_{p^{n-1}}\right)^{p \stackrel{i}{i}-\perp} \longrightarrow \mathscr{S}_{p^{n}}
$$


we see that

$$
\varphi_{n, 1}^{*} C(P)=C\left(R(Z / p)^{n-1}\right) \otimes \ldots \otimes C\left(R(Z / p)^{n-1}\right)
$$

since the total Chern class is multiplicative for sums of bundles.

12.

We may iterate the construction of (11.2) obtaining maps

$$
\varphi_{n, i}:\left[(Z / p)^{n-i}\right]^{p^{i}} \rightarrow \mathscr{S}_{p^{n}}
$$

Recall the theorem of $[4]$, see also [6, Section 3].

Theorem 12.2. The intersection of the kernels of the maps $p^{*}, i_{n-1}^{*}$ is zero.

Consequently, the images under the $\varphi_{n, i}^{*}$ of $C(P)$ completely characterise $C(P)$ and we have

$$
C\left(P \circ \varphi_{n, i}\right)=\stackrel{p}{i}^{i} C\left(R(Z / p)^{n-i}\right)
$$

13.

The constructions above and some obvious generalisations provide nearly complete information about the parts of $H^{*}\left(\mathscr{S}_{p^{n}}, Z / p\right)$ which map onto the pure polynomial pieces of the cohomology of the detecting groups in (12.1). However, there are many classes which map into classes involving the exterior algebras as well. These are much more complex and most of the work in [4] is involved in classifying them.

Some of them, though by no means all, can be obtained by a modification of the techniques above by lifting the stabilisation of the permutation representation in the diagram
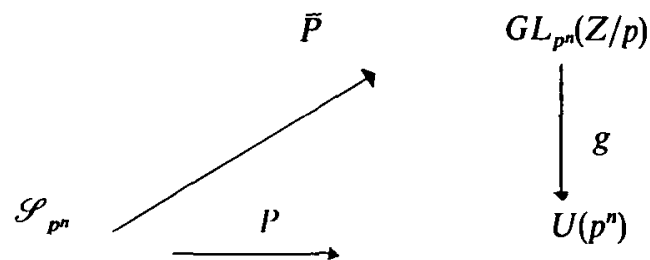

where $\bar{P}$ is the permutation representation in the modular group, and $g$ is the Green's representation [2]. Then use Quillen's calculation [7] of $H^{*}\left(G L_{n}(Z / q), Z / p\right)$ together with a slight extension of the techniques in Section 9 and Section 10, for appropriate $q$. 


\section{REFERENCES}

1. L. E. Dickson, A fundamental system of invariants of the general modular linear group with a solution of the form problem, Trans. Amer. Math. Soc. 12 (1911), 75-98.

2. J. A. Green, The characters of the finite general linear groups, Trans. Amer. Math. Soc. 80 (1955), 402-447.

3. S. Lang, Algebra (Addison Wesley, 1965).

4. B. M. MAnN, The cohomology of the symmetric groups, Trans. Amer. Math. Soc. 242 (1978), 157-184.

5. E. H. Moore, A two-fold generalization of Fermat's theorem, Bull. Amer. Math. Soc. 2nd series 2 (1896), 189-199.

6. D. Quillen, The Adams conjecture, Topology 10 (1971), 67-80.

7. D. Quillen, On the cohomology and $K$-theory of the general linear group over a finite field, Ann. Math. 96 (1972), 552-586.

8. N. E. Steenrod and D. B. A. Epstein, Cohomology Operations (Ann. of Math. Studies 50, Princeton U. Press, 1962).

9. B. M. MANN and R. J. Milgram. On the action of the Steenrod algebra, $\mathscr{A}_{(p)}$ on $H^{*}\left(M_{\mathrm{SPL}}, Z / p\right)$ at odd primes (preprint, Stanford University, 1976).

10. H. Mu, Modular invariant theory and cohomology algebras of symmetric groups, J. Fac. Sci. Univ. Tokyo 22 (1975), 319-369.

Bowdoin College

STANFORI University 